\title{
The Effects of Guide Pads on Bore Diameter Enlargement Magnitude in Deep Hole Drilling
}

\author{
Huang Zhang ${ }^{1}$, Xingquan Shen ${ }^{1}$, Yaoming $\mathrm{Li}^{1}$, Fuhe Yang ${ }^{1}$ and Paul Kwon ${ }^{2}$ \\ ${ }^{1}$ North University of China, Taiyuan 030051, China \\ ${ }^{2}$ Joincheer Mechanical \& Electrical Company, Korea
}

\begin{abstract}
Deep hole machining accuracy plays a crucial role in product's quality. The results of an investigation into the machining accuracy of deep drilling holes which highlights problems of bore diameter enlargement magnitude assessment are presented in this paper. Through the study of BTA deep drilling tool properties and mechanism, as well as the formation rule of deep hole surface, clarified the burnishing mechanism of guide pad in deep hole drilling, and obtained the directly relationship between surface formation rule and the guide pad. After drilling experimentrevealed the effect of guide pad on bore diameter enlargement magnitude.
\end{abstract}

\section{Introduction}

Deep-hole drilling is one of the most important processes for the production of a high-precision workpiece with high-quality holes [1]. The machining quality of deep-hole drilling has been studied for various machining conditions [2], [3]. Sakuma et al. [4] considered the behavior of the BTA solid boring system and postulated that the forming of a multi-corner hole is a certain kind of self-excited vibration. Deng et al. [5] used beam theory to investigate the hole straightness as affected by pilot bushing and intermediate support misalignments.

Literature investigation reveals that there are less rigorous theories to study the formation rule and its particularity of deep hole surface. And the guide mechanism of the guide paid as the most essential attributes of deep hole drilling is rarely studied. It's necessary to study the bore diameter enlargement magnitude, so as to improve the deep hole machining accuracy for strong support.

\section{The theoretical research of the diameter enlargement magnitude}

\subsection{Properties and mechanics of BTA drilling tool}

Deep hole drilling tools differ from conventional hole manufacturing tools due to a large length to diameter ratio, which have guide pads on the tool heads. In order to ensure the stability of drilling smoothly, the cutting forces are balanced by the reaction forces generated at the guide pads that are burnished on the inner hole surface, and consequently deep drilling tools are often referred to a 'self-piloting' tool, which allows high quality deep holes to be drilled. High pressure cutting fluids inject into the cutting region along the annular space between the drilling shaft and hole wall serving as coolant and lubricant at the same time, and flows over the cutting edge and returns with the cutting chips along the tool shaft inner hole, which is a kind of the inner ejection deep hole cutting machining method. Figure1 shows a typical deep drilling tool which has a single cutting edge. The outside corner of the cutting edge is in advance of the pads by a small distance $L_{L}$, so that they do not disrupt the cutting action.

Basedon the cutting force model of the deep holedrilling tools in Fig. 1, it can be seen intelligibly that the main cutting forces $P_{M A}, P_{M B}$ and $P_{M D}$ occurring at deep hole drill each blade are in the same direction that alltowardto the first guide pad, andthey are balanced by the reaction forces $P_{G 1}$ and $\mu \mathrm{P}_{\mathrm{G} 1}$ generated at the first guide pad. The radial cutting force component $P_{R D}, P_{R B}$ and $P_{R A}$ are balanced by the reaction forces $P_{G 2}$ and $\mu \mathrm{P}_{\mathrm{G} 2}$ generated at the second guide pad. At the same time, all guide pads are under the effect of friction torque, ultimately to ensure that the guide pads have stabilizing and guiding effects.

\subsection{The formation rule of deep hole surface}

To determine the direct relationship between the guide and the law of machining surface formation, B. J. Griffiths [6] using a specially designed quick stop device revealed features of the mechanics of the deep drilling process. The generation of white layer formations, as shown in Fig. 2, is the result of plastic deformation that generated by the two guide pads burnished the machined hole surface continuous periodically, and the high 
temperature can exceed $500^{\circ} \mathrm{C}$ in front of the pads, and combined with the rapid cooling effect caused by the pads revolving at high speed against the bore wall in the presence of the copious cutting fluid. Also it can be get

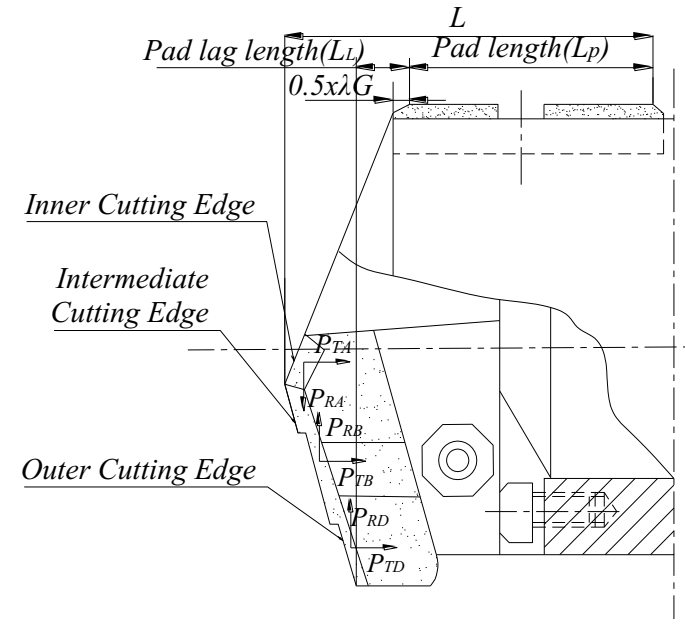

Figure 1. BTA drilling tool

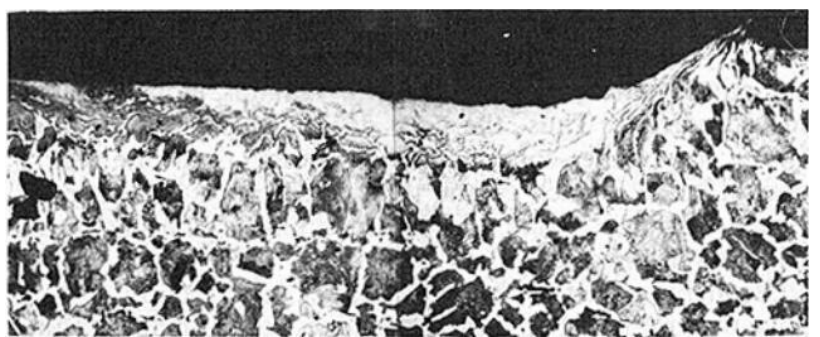

Figure 2. Microphotograph of burnishing region

The law of machining surface formation can be described as the ridge creation and modification occurs within the material burnished by guide pads in cycle, and the ridge truncation at the cutting edge land face, as shown in Fig. 3. Fig. 3(a) shows the hole form with the ridge burnished after the guide pads have passed, as the feed movement continue, the ridge has been truncated and area $\mathrm{ABC}$ removed in Fig. 3(b), when the tool has moved forward one feed rate, the area DEFG has been displaced by the guide pads, and in the forward direction, the new ridge $\mathrm{HIG}$ recreated and in the backward direction the plastic flow tends to fill in the previous indentation area KLEM.

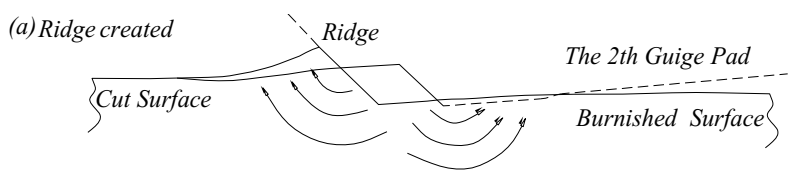

(b) Ridge truncated

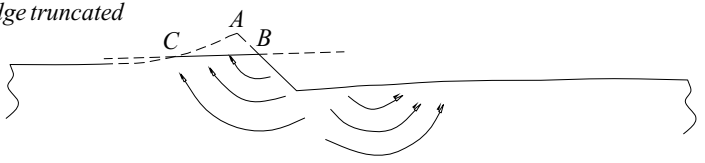

(c) Ridge recreated \I

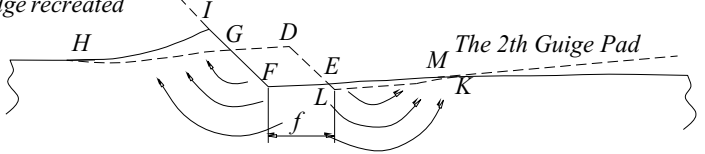

Figure 3. Schematic representation of deformation cycle that the hole diameter at the cut surface is less than at the burnished surface, which implying that considerable plastic flow has occurred.

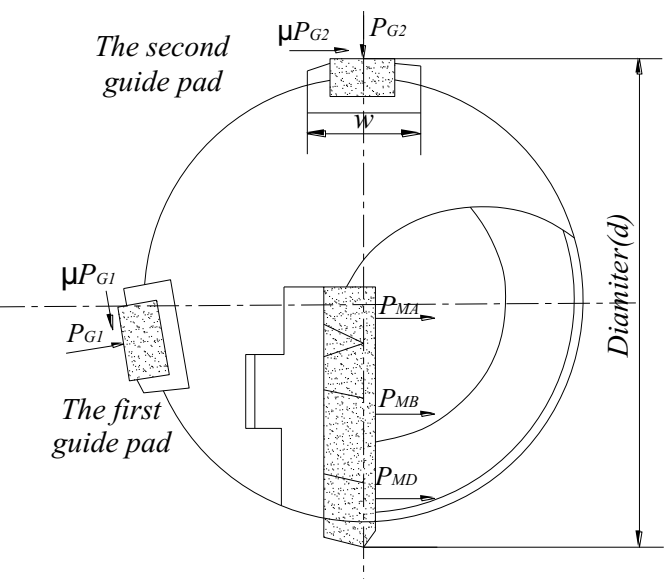

\subsection{The affect factors of deep hole bore diameter enlargement magnitude}

Comprehensive above research, the plastic deformation and elastic deformation are created simultaneously after the guide pads burnished, that lead to the enlargement of the machined hole. With the different workpiece materials, the plastic deformation part of the hole wall is differentand proportional to feed rate $f$. The deep hole bore diameter enlargement magnitude $\Delta \mathrm{D}$ [7] caused by guide pads brunished can be represented astheequation (1):

$$
\Delta \mathrm{D}=\left(\frac{\mathrm{K}_{\mathrm{S}} \cdot \mathrm{D}}{2 \mathrm{HB} \cdot \mathrm{w}}-\mathrm{C}+\frac{1}{4}\right) \mathrm{f} \cdot \tan \lambda_{\mathrm{G}}
$$

where $K_{S}$ is the specific cutting force of workpiece material $\left(\times 9.8 \mathrm{~N} / \mathrm{mm}^{2}\right), \mathrm{D}$ is the hole diameter, $\mathrm{HB}$ is the brinell hardness of the workpiece, $\mathrm{w}$ is the width of the guide pad, $\mathrm{C}$ is the ratio of the feed rate to the actual length of the guide pad fore-end and hole wall contact surface, $\lambda_{G}$ is the entrance angle of guide pad, as shown in Fig. 1. It is not difficult to draw that the thinnerhole wall, the bigger deformation, and the hole shape error will be produced.

In addition, The influencing factors of bore diameter enlargement also includes the following aspects:the cutting heat causes the material heat bilges cold shrink, the extension ofthe built-up edge on the outer edge cause the expansion of the aperturein the processing of drilling, and if the corner of outer cutting edge is blunt, it will generate elastic deformation and cutter relieving in the drilling region, that further cause the narrowing of the bore diameter.

\section{Drilling experimental}

To examine the effects of guide pads, a fundamental experiment of a BOTEK deep hole tools with diameter of 
$30 \mathrm{~mm}$ was made, and the correlative parameters are as follows: the width $\omega$ of guide pad is $7.9 \mathrm{~mm}$, the entrance angle $\lambda_{G}=10^{\circ}$, rotating rate $\mathrm{n}$ of workpiece is630 $\mathrm{r} / \mathrm{min}$, the workpiece materials are $\mathrm{A} 2017-\mathrm{T} 4(\mathrm{HB}=98)$ and $\mathrm{A} 2017-0(\mathrm{HB}=51)$, and assigning a value to $\mathrm{C}$ is 2 , also the feed rate can be adjusted from 0 to $0.2 \mathrm{~mm} \cdot \mathrm{r}^{-1}$.

(a)

(b)

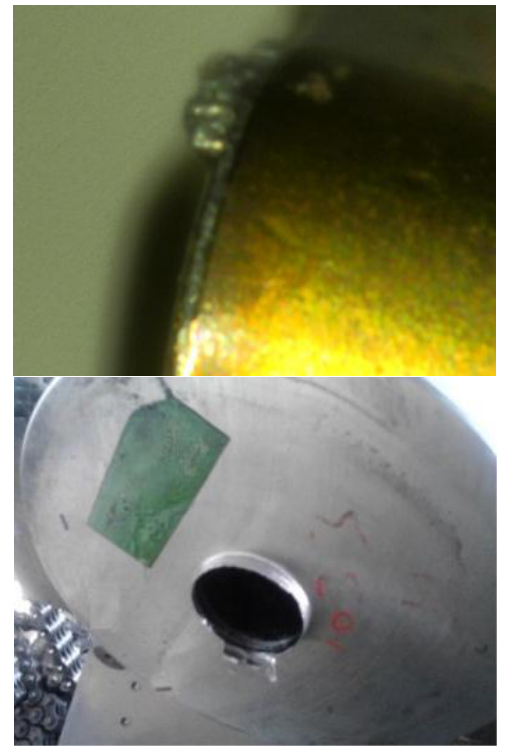

Figure 4. The effects of guide pad burnished, (a) and (b) respectively represent the guide paid and the machined hole

(a)

(b)

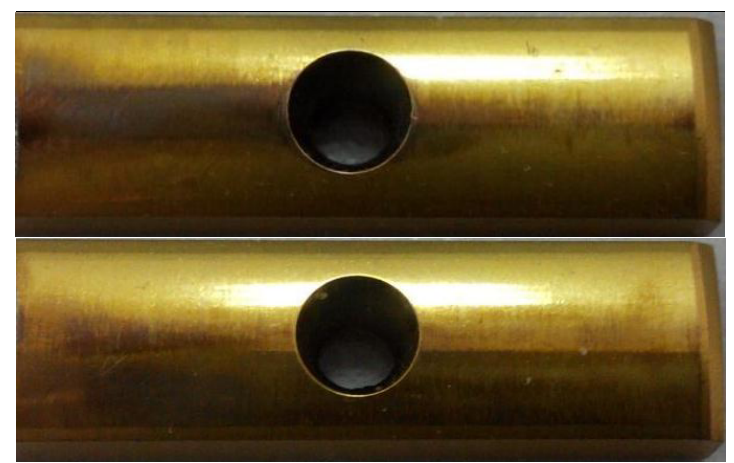

Figure 5. The wear state of guide pad, (a) and (b) respectively represent the first and second guide pad

The deep hole formed characteristics and wear state of guide pad are obtained by high depth of field microscope shown respectively in Fig. 4-Fig. 5, and comparatively analyzed the influence rules of deep hole bore diameter expanding quantity through adjusting feed rate. As shown in Figure 4, a matter worthy of reflection, the residual workpiece material after being squeezed is stored on the guide pad entrance, and at the machined hole exit with a redundant circular workpiece after the drilling is finishing. These are the characterization of the ridge creation after the guide paid passing. These results are very encouraging in that guide pads played an important role in the hole surface formation regularity, and enlarge influence to the machined surface simultaneously. In addition, Fig. 5 shows the wear states of first and second guide pad respectively. It can be seen that the wear state with coating peelingon the front end locale of guide pad is more serious than the back-end locale, and the wear stateon the first guide pad presented a dark brown is more serious than the second guide pad. That is mainly because the second guide pad is lagged behind the first guide pad about $\mathrm{f} / 4$. Also it can be come to the conclusion that the stress of the first guide paid is significantly bigger than the second guide paid which lead to the first guide paid is wear more serious through studying the cutting force model of the deep hole drilling tools in Fig. 1.

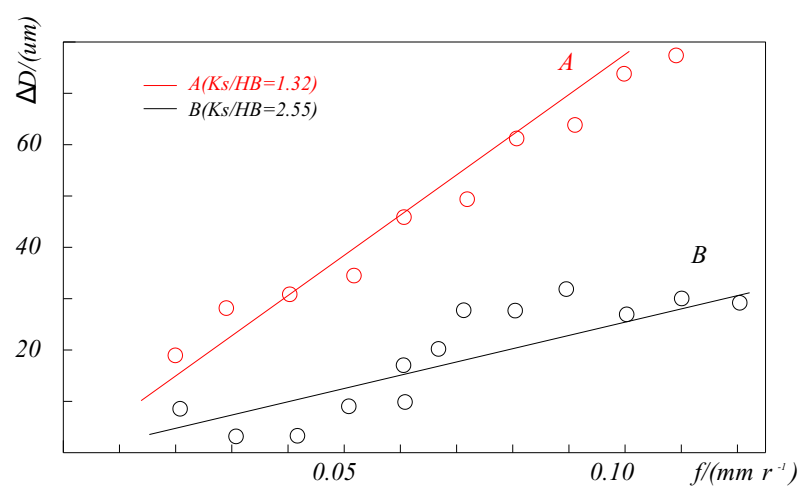

Figure 6. The changes of bore diameter enlargement magnitude along with feed rate

Fig. 6 shows the changes of bore diameter extensive magnitude along with feed rate under the condition of $K_{s} / \mathrm{HB}$ are 1.32 and 2.55 respectively. In the case of other factors unchanged, with the increase of the feed rate, the bore diameter extensive magnitude also increases accordingly. Combined with the Equation (1), because the $K_{S}$ is associated with the strength and toughness of workpiece materials, while the HB value is related to the material heat treatment conditions, so in general, the bore diameter extensive magnitudeis remarkable for higher strength, toughness and without hardening material. It is not hard to analyze that no matter what kind of material, to increase feed rate will cause the proportional increase of bore diameter enlargement magnitude.

\section{Conclusions}

Through the description of the deep hole drilling process, revealed the formation of machining surface by BTA deep hole drilling tools, and put forward the root causes for the enlargement of the machined hole, that both the cutting stage and the burnishing stage, make the machined inner hole surface produce plastic deformation several times.

The influencing factors of bore diameter enlargement magnitude have been studied. Due to the bore diameter expanding and reducing factor is not unilaterally, it is conceivable that the actual diameter depends on the comprehensive effect of various factors, while the bore diameter enlargement magnitude will proportionally increase along with the increase feed rate. Ultimately, the bore diameter enlargement magnitude is directly related to the guide pad.

Combined with the mechanical analysis of BTA drilling model and the drilling experiments of different hardness materials, researched the wear state of guide pad and the deep hole formation characteristics, verified the burnishing effect of guide pads and the root causes of the machined hole enlargement. 


\section{Acknowledgements}

This work was financially supported by the National Natural Science Foundation of China (51175482) and International S\&T Cooperation Program of China (2013DFA70770).

\section{References}

[1] CS Deng, JH Chin. Roundness Errors in BTA Drilling and a Model of Waviness and Lobbing Caused by Resonant Forced Vibrations of Its Long Drill Shaft. Journal of manufacturing Science and Engineering, 126: 524-533 (2004).

[2] Aized T, Amjad M. Quality improvement of deep-hole drilling process of AISI D2 [J]. The International Journal of Advanced Manufacturing Technology, 69(9-12): 2493-2503 (2013).

[3] Matsuzaki K, Ryu T, Sueoka A, et al. Theoretical and experimental study on rifling mark generating phenomena in BTA deep hole drilling process (generating mechanism and countermeasure) $[\mathrm{J}]$. International Journal of Machine Tools and Manufacture, 88: 194-205 (2015).

[4] Sakuma K, Taguchi K, Katsuki A, et al. Self-guiding action of deep-hole-drilling tools [J]. CIRP Annals-Manufacturing Technology, 30(1): 311-315(1981).

[5] Deng, C. S., Huang, J. C., and Chin, J. H., "Effects of Support Misalignments in Deep-Hole Drill Shafts on Hole Straightness,' Int. J. Mach. Tools Manuf., 41(8): 1165-1188 (2001).

[6] Griffiths B J. Modelling complex force systems, Part 1: The cutting and pad forces in deep drilling [J]. Journal of engineering for industry, 115(2): 169-176 (1993).

[7] Jun Wang. Modern deep processing technology [M]. Harbin: Harbin Institute of Technology Press, 2 (2005). 